\title{
Fibronectin as an opsonic regulator of monocyte phagocytosis
}

\author{
AW SIMPSON, BJ BOUGHTON \\ From the Haematology Department, University of Birmingham, Edgbaston, Birmingham
}

SUMMARY Experiments using human monocytes and the yeast Saccharomyces cerevisiae have shown that fibronectin is a major plasma opsonin. Further studies have shown that fibronectin promotes the ingestion as well as the adherence of micro-organisms. These observations are independent of the non-physiological concentrations of heparin used in other assay systems.

Evidence from rat liver slice and macrophage monolayer assays suggests that plasma fibronectin is a major opsonic regulator of fixed macrophage function. ${ }^{1-4}$ These assays, however, use target particles coated with gelatin and depend to a varying degree on the presence of heparin. We have recently developed an opsonin assay which utilises human peripheral blood monocytes and the micro-organism Saccharomyces cerevisiae. We have used this assay to examine the effect of plasma fibronectin on the phagocytosis of micro-organisms by human monocytes.

\section{Material and methods}

Human plasma was obtained from blood drawn into $3.13 \% \mathrm{wt} / \mathrm{vol}$ trisodium citrate, and plasma fibronectin was prepared by affinity chromatography on gelatin Sepharose and arginine Sepharose. Opsonic activity was assayed by electronic particle size analysis of human monocytes and the yeast $S$ cerevisiae as previously described. ${ }^{\circ}$ In addition, ingested and adherent yeasts were visually distinguished by staining with toluidine blue and Procion rubine $\mathrm{MX}$ dye. With this technique, ingested and adherent yeasts are coloured red and blue respectively.? Heparin was not added to either of these two assays.

Fibronectin free plasma was obtained by applying $10 \mathrm{ml}$ citrated plasma to a $10 \mathrm{ml}$ column of gelatin Sepharose $4 \mathrm{~B}$, equilibrated with $50 \mathrm{mM}$ Tris $\mathrm{HCl}$ $\mathrm{pH} 7 \cdot 5$. The eluate was concentrated by ultrafiltration to its original volume using fibrinogen concentration as a guide. ${ }^{8}$ Fibronectin antigen concentrations were assayed by immunoelectrophoresis using a monospecific polyclonal sheep antihuman fibronectin antibody. ${ }^{4}$ In order to inhibit activation of the $\mathrm{C}_{3}$ component of complement, dipotassium edetic acid was added to plasma to a final concentration of $20 \mathrm{mM}$. Purified human IgG $(14 \mathrm{~g} / \mathrm{l})$ was obtained from the Immuno Diagnostic Research Laboratory at Birmingham University.

The statistical significance of differences between mean values was assessed by Student's t test.

\section{Results}

Fibronectin concentrations in citrated plasma from 30 normal healthy subjects gave a range (mean \pm 2 SD) of $254-501 \mathrm{mg} / \mathrm{l}$. In all of the following experiments fibronectin was used in concentrations which did not exceed the range in normal plasma.

Fig. 1 shows the stimulation of phagocytosis by increasing concentrations of purified fibronectin and

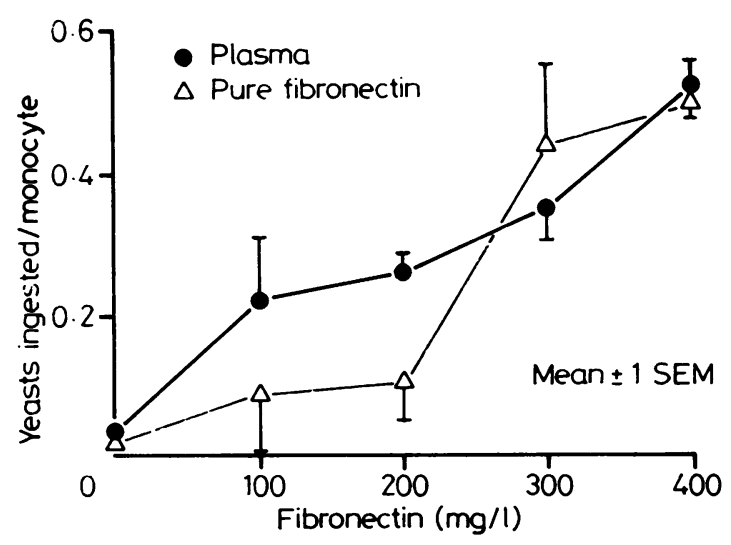

Fig. 1 Monocyte uptake of yeasts after opsonisation with increasing concentrations of fibronectin or whole plasma. 


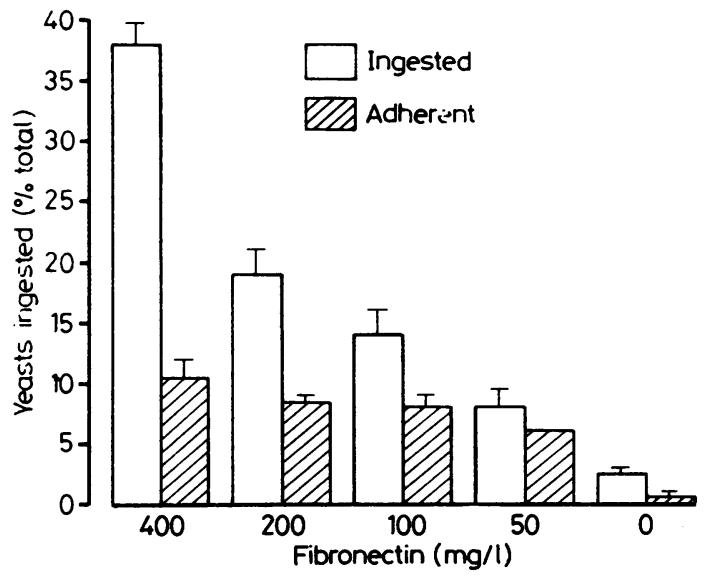

Fig 2 Percentage of yeasts ingested or adhering to monocytes after opsonisation with increasing concentrations of purified fibronectin. Mean value $\pm 1 S E M$ for each concentration is shown. Hatched areas represent the adherent yeasts which have not yet been ingested.

whole plasma. A dose response was seen in both cases and the mean values for opsonisation at each fibronectin concentration were not significantly different $(\mathrm{p}>0 \cdot 1)$.

Fig. 2 shows a visual assessment of yeast adherence and ingestion in response to increasing fibronectin concentrations. In the absence of fibronectin no significant adhesion or ingestion of yeasts was observed, but these were considerably stimulated by increasing concentrations of fibronectin. These results show clearly that higher fibronectin concentrations stimulate the ingestion of a higher proportion of adherent organisms.

The relative importance of fibronectin as an opsonin is shown in Fig. 3. Fibronectin accounted for $60 \%$ of the opsonic activity of citrated plasma, and most of the activity in fibronectin free plasma was due to IgG. When purified IgG was used with fibronectin as an opsonin the combined effect appeared additive and no synergy was observed. Inhibition of complement with $20 \mathrm{mM}$ edetic acid reduced opsonic activity by only $10 \%$. A fibronectin free preparation of human albumin $(35 \mathrm{~g} / \mathrm{l})$ was used to examine non-specific protein effects and this showed no opsonic activity.

\section{Discussion}

The uptake of $S$ cerevisiae opsonised with purified fibronectin or whole plasma showed a clear dose response. The experiments with dyed yeast showed that purified fibronectin promoted the ingestion of

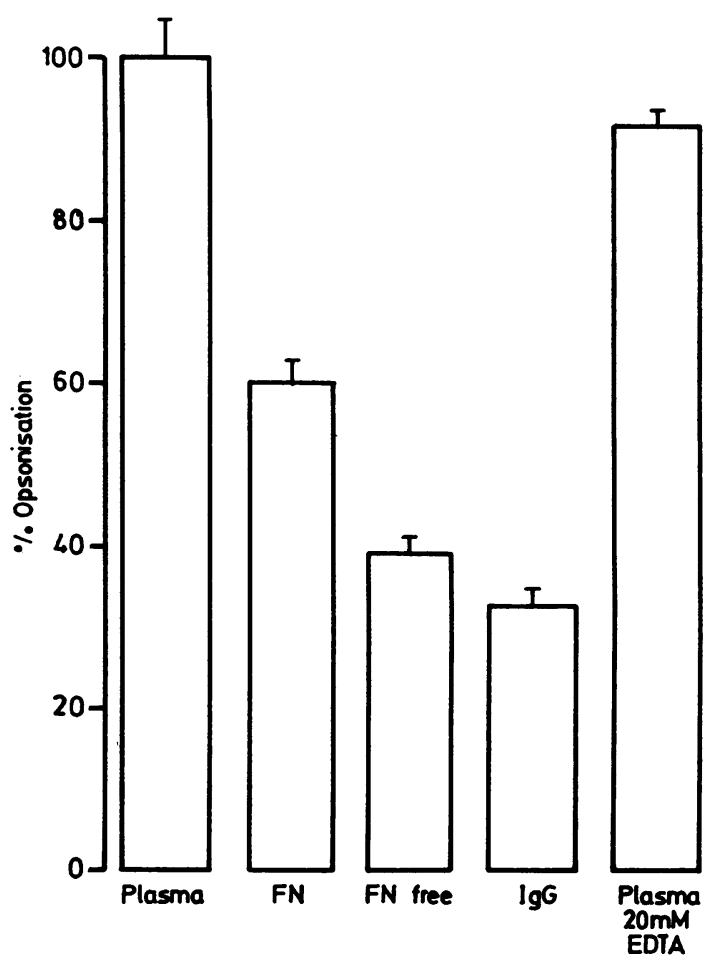

Fig. 3 Opsonic effect of purified fibronectin (FN), whole citrated plasma, fibronectin free plasma, purified IgG, and EDTA plasma. Plasma samples from the same donor pool and purified fibronectin were used at a concentration of $400 \mathrm{mg} / \mathrm{l}$. No fibronectin could be detected in fibronectin free plasma samples or in the purified IgG. Opsonisation is shown as a percentage of the activity shown by citrated plasma, and values are indicated as a mean $\pm 1 S E M$.

micro-organisms by monocytes in addition to its effect as an adhesion promoter. These findings contrast with those obtained with gelatinised target particles and monocyte monolayers, in which adhesion but not the subsequent ingestion of particles could be dẹmonstrated. ${ }^{10}$

Since fibronectin does not bind to certain Gram negative organisms and in some assay systems has $\sigma$ not promoted the ingestion of particles by human $N$ monocytes, it has been suggested that fibronectin $\mathrm{C}_{\mathrm{W}}$ may not be an important opsonin." Our own studies suggest, however, that it accounts for most of the non-specific opsonic activity of plasma. The absence of opsonic activity in fibronectin free albumin excluded the possibility of non-specific protein effects.

Heparin is essential for the uptake of gelatinsed target particles in the rat liver slice assay. ${ }^{3} \mathrm{~A}$ rat peritoneal macrophage assay is also, to some extent, 
heparin dependent. ${ }^{4}$ Our own experiments with human monocytes were conducted without heparin present, but we have found that the opsonic activity of plasma was enhanced by heparin. The activity of purified fibronectin was greatly reduced, however, and this is the subject of further investigations.

We are indebted to the West Midland Regional Blood Transfusion Service for buffy coat preparations and to the Department of Immunology, Birmingham University, for the gift of the Procion rubine MX dye. Dr BJ Boughton is a Leukaemia Research Fund Senior Lecturer in Haematology. We thank Miss Catherine Garvey for preparing the manuscript.

\section{References}

' Saba TM, Blumenstock FA, Scovill WA, Bernard H. Cryoprecipitate reversal of opsonic $\alpha_{2}$-surface binding glycoprotein deficiency in septic, surgical and trauma patients. Science 1978;201:622-4.

${ }^{2}$ Scovill WA, Saba TM, Blumenstock FA, Bernard H, Powers SR Jr. Opsonic $\alpha_{2}$-surface binding glycoprotein therapy during sepsis. Ann Surg 1978;188:521-9.
${ }^{3}$ Saba TM, Filkins JP, Di Luzio NR. Properties of the "opsonic system" regulating in vitro hepatic phagocytosis. J Reticuloendothelial Soc 1966;3:398-404.

${ }^{4}$ Gudewicz PW, Molnar J, Ming Zong Lai, et al. Fibronectin mediated uptake of gelatin coated latex particles by peritoneal macrophages. J Cell Biol 1980;87:427-33.

${ }^{5}$ Vuento M, Vaheri A. Purification of fibronectin from human plasma by affinity chromatography under non-denaturing conditions. Biochem J 1979; 183:331-7.

- Simpson AW, Boughton BJ. A human monocyte system for the assay of plasma opsonins. J Immunol Methods 1983;63:321-7.

' Shaala AY, Dhaliwal HS, Bishop S, Ling NR. Ingestion of dyed-opsonised yeasts as a simple way of detecting phagocytes in' lymphocyte preparations. Cytophylic binding of immunoglobulins by ingesting cells. J Immunol Methods 1979; 27: 175-87.

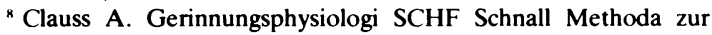
Bestimmung des Fibrinogens. Acta Haematol 1957;17:32746.

${ }^{4}$ Laurell CB. Electroimmuno assay. Scand J Clin Lab Invest 1972;29(suppl 124):21-37.

${ }^{10}$ Bevilacqua MP, Amrani DL, Mosesson MW, Bianco C. Binding of cold-insoluble globulin (plasma fibronectin) on human monocytes. J Exp Med 1981;153:42-60.

" Doran JE. A critical assessment of fibronectin's opsonic role for bacteria and microaggregates. Vox Sang 1983;45:317-48.

Requests for reprints to: Mr AW Simpson, Haematology Department, The Queen Elizabeth Hospital, Queen Elizabeth Medical Centre, Edgbaston, Birmingham B15 2TH, England. 